\title{
Phase calibration approaches for radar interferometry and imaging configurations: equatorial spread $F$ results
}

\author{
J. L. Chau ${ }^{1}$, D. L. Hysell ${ }^{2}$, K. M. Kuyeng ${ }^{1}$, and F. R. Galindo ${ }^{1}$ \\ ${ }^{1}$ Radio Observatorio de Jicamarca, Instituto Geofísico del Perú, Lima \\ ${ }^{2}$ Earth and Atmospheric Sciences, Cornell University, Ithaca, NY, USA
}

Received: 25 September 2007 - Revised: 16 April 2008 - Accepted: 29 April 2008 - Published: 5 August 2008

\begin{abstract}
In recent years, more and more radar systems with multiple-receiver antennas are being used to study the atmospheric and ionospheric irregularities with either interferometric and/or imaging configurations. In such systems, one of the major challenges is to know the phase offsets between the different receiver channels. Such phases are intrinsic to the system and are due to different cable lengths, filters, attenuators, amplifiers, antenna impedance, etc. Moreover, such phases change as function of time, on different time scales, depending on the specific installation. In this work, we present three approaches using natural targets (radio stars, meteor-head and meteor trail echoes) that allow either an absolute or relative phase calibration. In addition, we present the results of using an artificial source (radio beacon) for a continuous calibration that complements the previous approaches. These approaches are robust and good alternatives to other approaches, e.g. self-calibration techniques using known data features, or for multiple-receiver configurations constantly changing their receiving elements. In order to show the good performance of the proposed phase calibration techniques, we present new radar imaging results of equatorial spread F (ESF) irregularities. Finally we introduce a new way to represent range-time intensity (RTI) maps color coded with the Doppler information. Such modified map allows the identification and interpretation of geophysical phenomena, previously hidden in conventional RTI maps, e.g. the time and altitude of occurrence of ESF irregularities pinching off from the bottomside and their respective Doppler velocity.
\end{abstract}

Keywords. Ionosphere (Equatorial ionosphere; Ionospheric irregularities; Instruments and techniques)

Correspondence to: J. L. Chau

(jchau@jro.igp.gob.pe)

\section{Introduction}

Radar systems with multiple-receiver antennas have significantly improved the studies of atmospheric and ionospheric irregularities and also the accuracy and precision of their derived parameters. Woodman (1971) was the first to use an interferometer configuration with two receiving antennas to study the inclination of the magnetic field at ionospheric altitudes over the Jicamarca Radio Observatory (JRO). Later Farley et al. (1981) introduced the radar interferometry technique to study field-aligned ionospheric irregularities. Since then, the technique using two (for one dimension) or three non-collinear (for two dimensions) antennas has been used to study different ionospheric and atmospheric irregularities at different sites across the World.

For example, in the case of equatorial ionospheric irregularities their zonal velocity can be derived using a two antenna interferometer (e.g. Kudeki et al., 1982; Hysell and Burcham, 1998). In the case of atmospheric research, spatial interferometry has been used to determine the mean offvertical position of the atmospheric aspect sensitivity function, of particular importance for the accurate determination of the mean vertical wind (e.g. Chau and Balsley, 1998, and references therein). In the latter application, receiver phase offsets need to be properly taken into account. In the majority of other applications, using single baseline interferometry, knowing the receiver phase offsets is not so critical, since only the relative echo location is needed (e.g. to derive the zonal drift).

Kudeki and Sürücü (1991) extended the radar interferometry technique to use more receivers, allowing the measurement of more than one scattering region inside the illuminated volume. The number of scattering regions that can be detected with a given configuration depends on the number of degrees of freedom of the interferometer configuration, i.e. the number of non-redundant antenna separations. Such technique is now called radar imaging or aperture synthesis

Published by Copernicus Publications on behalf of the European Geosciences Union. 
radar imaging and has been improved and applied at different altitudinal and latitudinal regions in the last decade. For example, it has been used to study equatorial, midlatitude and high latitude ionospheric irregularities (e.g. Hysell, 1996; Hysell et al., 2002, 2004a; Chau and Hysell, 2004; Hysell et al., 2004b; Bahcivan et al., 2006) and lower atmospheric irregularities (e.g. Chau and Woodman, 2001; Palmer et al., 1998). For more details on the radar imaging technique we recommend reading Woodman (1997) and Hysell and Chau (2006).

In radar imaging, taking into account the phase offsets between receiving channels is crucial for the technique to work. Such phase offsets are intrinsic to the receiving system and could be due, among other factors to differences in: cable lengths, filter responses, connectors, etc. Moreover, these offsets can change on different time scales, e.g. months to years for cable aging, or within few seconds due to hardware changes (e.g. filter, attenuators, cables) or hardware failures (e.g. loose connections). Depending on the application, one might need an absolute phase calibration, e.g. when comparing the radar results against other observations like in-situ observations from rockets or satellites (e.g. Bahcivan et al., $2005,2006)$. On some occasions only relative phase calibrations are needed, for example to determine the motion and morphology of large scale irregularities (e.g. Hysell et al., 2004a; Chau and Hysell, 2004).

In specular meteor radar (SMR) systems using interferometry, knowing the absolute phase offsets is very important. Even though those systems are after single scattering regions in the illuminated volume, nowadays 5 antennas are used to improve the precision and to remove angular ambiguity (e.g. Jones et al., 1998; Holdsworth et al., 2004a), allowing a good determination of the mesospheric winds and the height distribution of specular meteor echoes.

Given the importance of determining the phase offsets between receivers, many authors have proposed different calibration approaches with varying degrees of performance depending on the interferometer system and the application. Here is a brief summary listing some of the calibration techniques proposed in the literature:

- Feeding a common signal at the input of each receiver line or close to the interferometric system. This technique, and its variants, works well for receiving antennas that are closely spaced or for systems where the common signal is fed through cables of equal (or known lengths) that are held at a constant temperature. The former is usually the case for meteor systems, while the latter usually requires expensive and static configurations. Valentic et al. (1997) used a similar approach, but instead of injecting a common signal, they used a radio beacon from a known location. By moving the radio beacon around, in addition to the phase offsets, they were able to determine the actual location of the receiving antennas.
- Use of radio stars. Woodman (1971) and Palmer et al. (1996) used the radio signals from Hydra A and Cygnus A to calibrate the Jicamarca and MU radar interferometer systems, respectively. The procedure allows a very precise calibration, but it only works at a time when ionospheric scintillation are weak or absent (few days a year, depending on the site location). Moreover, such calibration can be used only during few minutes a day.

- Self-calibration using known data features. Chau and Woodman (2001) used the known averaged spatial characteristics of equatorial electrojet (EEJ) irregularities. The echoes from these irregularities come from the locus of perpendicularity in the meridional direction and from the center of the transmitting beam in the zonal direction. Similarly, Holdsworth et al. (2004b) used the known statistical distribution of specular meteor echoes to calibrate SMR systems. Hysell and Woodman (1997) and Hysell and Chau (2002) found the relative phase differences by minimizing the peaks of the estimated image. This procedure was applied by incorporating one antenna at the time to the minimization procedure.

In all these cases, the calibration procedure was done on a campaign basis and some of them are very time demanding. As mentioned above, unless the receiving system is static and very stable, a systematic procedure is needed.

In the following sections we present three different approaches that allow either absolute or relative phase calibrations. In the case of the relative calibration, although useful for some applications, we also present possible solutions to determine the absolute calibration. Examples of equatorial electrojet (EEJ) images are presented to describe the performance of the technique. One of the techniques also uses the radio star Hydra, but in this work we present two different approaches and a general formulation for a system with any number of receiving antennas. We also present an approach using meteor-head and meteor-trail echoes. The former is only applicable on systems with very high sensitivity, while the latter can be used in low power systems. Then we present a solution for JRO's calibration procedure that combines the benefits of the previous techniques with the need of having a continuous phase calibration procedure. Having such a solution, allows a facility like JRO where receiving components are constantly changing, to be able to apply imaging techniques in an operational fashion (JULIA-like modes) (e.g. Hysell and Burcham, 1998).

In the last two sections we present and discuss the radar images of equatorial spread $\mathrm{F}$ (ESF) irregularities obtained with the previous phase calibration techniques. The interpretation of these radar images are accompanied by the interpretation of a modified range-time intensity (RTI) map color coded with the Doppler information (RTDI). The proposed RTDI allows the identification of geophysical features previously hidden in typical RTI maps. For example, we show, 
by comparing against the radar images, that ESF irregularities pinching-off from the bottomside can be identified and characterized with the proposed RTDI.

\section{Phase calibration approaches}

In Fig. 1 we show a picture of the Jicamarca main antenna along with an imaging configuration example using 8 receiving antennas denoted by the square modules in red. The circle in yellow denotes the position of a radio beacon on one of the hills surrounding Jicamarca. The use and the importance of the beacon will be described later.

The Jicamarca multiple-receiver antenna can be arranged in a variety of ways depending on the application. For example on some occasions, pairs of modules have been combined to form separate receiving antennas, or smaller Yagi arrays have been used to allow for more antenna baselines with shorter separations. Note that with the latter examples there could be antenna modules with different antenna patterns. Hysell and Chau (2006) improved the Maximum Entropy (MaxEnt) imaging algorithm to allow for receiving antennas with different antenna patterns.

In the following sections, for all practical purposes, all the sources used for phase calibration can be considered as pointtargets, particularly for the frequencies of interests (VHF) and the antenna sizes involved $(10-150 \mathrm{~m}$ on each side). Therefore, the expected phase difference between signals received at antennas $i$ and $j$ due to a point target located at directions cosines $\theta_{x}$ and $\theta_{y}$ is given by

$\phi_{i j}=\frac{2 \pi}{\lambda}\left(\Delta x_{i j} \theta_{x}+\Delta y_{i j} \theta_{y}+\Delta z \theta_{z}\right)+\Delta_{i j}$

where $\theta_{z}=\sqrt{1-\theta_{x}^{2}-\theta_{y}^{2}}, \Delta x, \Delta y, \Delta z$ are the separations (in $\mathrm{m})$ between antennas $i$ and $j$ in the $x, y, z$ directions respectively, $\lambda$ is the radar wavelength (in $\mathrm{m}$ ), and $\Delta_{i j}=\delta_{i}-\delta_{j}$. The phase offsets for receivers $i$ and $j$ are $\delta_{i}$ and $\delta_{j}$ respectively. To simplify the formulation, we assume that all antennas are in the same horizontal plane, so $\Delta z=0$ for all antenna pairs.

Similarly the complex cross-correlation function between signals at antennas $i$ and $j$ due to a point target is given by

$\rho_{i j}=A_{i j} \exp \left[\imath k\left(\Delta x_{i j} \theta_{x}+\Delta y_{i j} \theta_{y}\right)+\imath \Delta_{i j}\right]$

where $k=2 \pi / \lambda$ and $A_{i j}$ is the coherence magnitude for antenna pair $i j$. For targets coming from overhead, $A_{i j}$ is usually constant for all pairs, particularly if the antenna patterns of receiving antennas are the same and the target is very narrow.

In both cases, the problem consists of finding the receiver phase offsets by measuring the phase difference and/or crosscorrelation between antennas. In the case of the example with 8 receiving antennas, there are 7 unknowns (we can always assume one of the offsets to be zero) and 28/56 degrees of freedom if the phases/correlations are used.

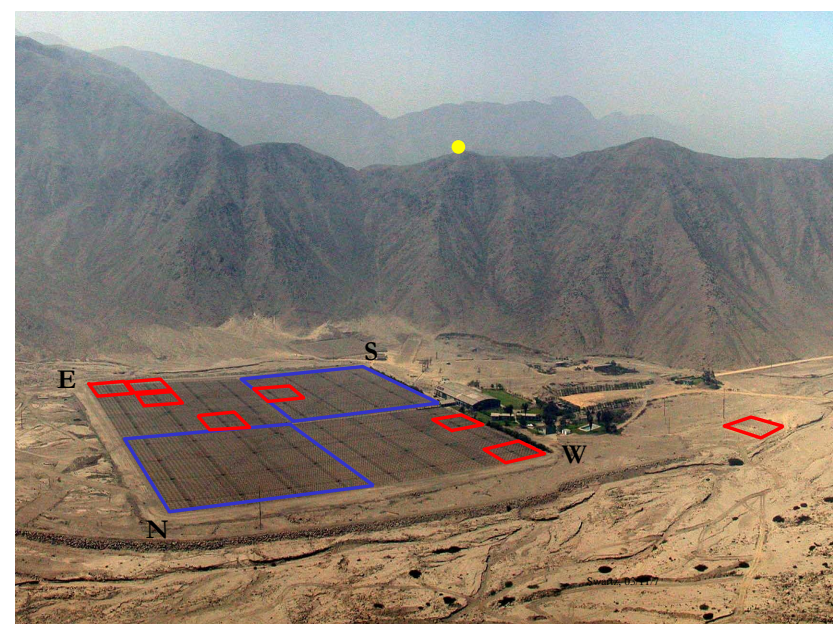

Fig. 1. Example of a radar imaging configuration at Jicamarca using 8 receiving antennas (in red). The transmitting antenna is denoted in blue. For this particular example, all the possible antenna separations are non-redundant in the zonal (east-west) direction. The yellow circle represents the location of the radio beacon on one of the hills.

\subsection{Absolute phase calibration using the Hydra radio star}

Radio stars are good point sources with known locations. Depending on the site location and frequency of operation, there could be one or more radio stars with enough intensity as to be used for calibration purposes. For example, Palmer et al. (1998) used Cygnus A that is visible over the MU radar in Japan. In the case of Jicamarca, we can observe the Hydra A radio star $\left(9 \mathrm{~h} 17^{\prime} \mathrm{RA}, 12^{\circ} 5^{\prime} \mathrm{Dec}\right)$. Such signal was first used by Woodman (1971) to calibrate a two-receiver interferometer using quarter sections. In the case of Hydra, its power intensity can be easily detected using antenna quarter sections. Using modules (1/64th of the main antenna), the Hydra intensity is very weak given the wider antenna beam pattern, i.e. allowing contribution from other radio sources in the sky. Its detection depends on the number of independent samples used. However, the phase of cross-correlations between different antennas is very precise and accurate. Although the Hydra signal itself is of similar magnitude than the background skynoise, the former is very narrow and localized in space, therefore it is easily detectable from crosscorrelating signals of different antennas.

As mentioned above, radio star signals have to be used during times when ionospheric scintillations are expected to be weak or absent. In the case of Jicamarca, one has to avoid times when ESF and strong EEJ irregularities are expected. In the latter case, Woodman et al. (1995) has shown that Hydra scintillations due to EEJ irregularities are strong enough to measure features of the kilometer-scale EEJ waves.

Since radio stars are located at infinity, their signal is independent of range, and one could use such features to decrease 
a) $\mathrm{AOA}$ with respect to baseline

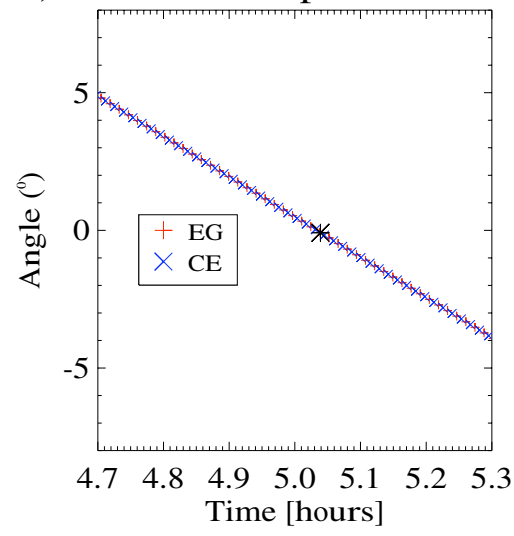

b) Measured AOA

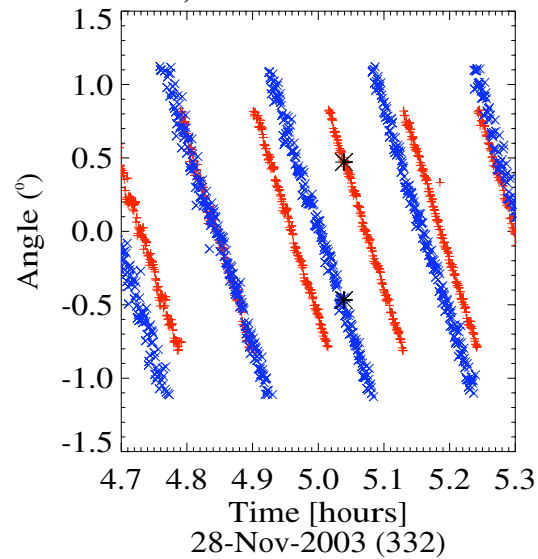

c) Residual Phase

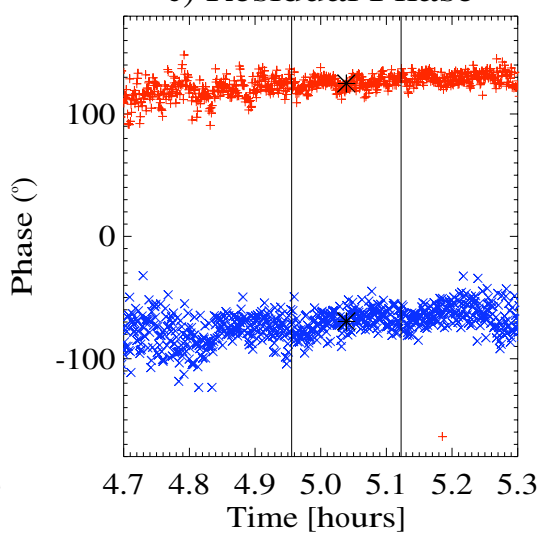

Fig. 2. Fringe stopping. (a) Expected Hydra angle of arrival (AOA) with respect to a baseline; (b) Measured AOA (note phase wrapping due to $2 \pi$ ambiguity), and (c) Residual phase after removing the expected Hydra information. The star symbol in panel (b) indicates the transit time over Jicamarca. The vertical lines in panel (c) indicate the time interval for getting an average value ( \pm 5 min around Hydra's transit time). Red $(+)$ and blue $(\times)$ indicate values for a selected pair of antennas, i.e. EG and CE.

the statistical uncertainty by averaging in range. Moreover, one can improve the averaging process further by taking into account the Earth's rotation, as the radio star transits over the radar site. Below we present two approaches: one that deals with just the phase information and one that works with both phase and amplitude (or real and imaginary components). Similar techniques have been used by radio astronomers and are called fringe stopping and fringe fitting, respectively (e.g. Taylor et al., 1999).

\subsubsection{Fringe stopping}

As mentioned above, the expected signal of a point target source could be represented by the phase difference or the cross-correlation between antennas. Given that the radio star is very narrow, one can work only with the phase information. However, in order to decrease the influence of the background sky, convolved with the antenna pattern, we recommend using at least $N-1$ antenna pairs that are separated by at least two times the dimensions of the largest antenna, where $N$ is the number of receiving antennas.

In Fig. 2 we show an example of the application and performance of the fringe stopping procedure. Figure 2a shows the expected angle of arrival (AOA) (in degrees) of the Hydra signal with respect to a given baseline as function of time for 2 selected baselines: EG (red, + ) and CE (blue, $x$ ) . The star symbols (in black) indicate the transit time of Hydra over Jicamarca. Figure $2 b$ shows the measured AOA for the same antenna pairs used in panel (a). Given the $2 \pi$ ambiguity of the phase estimator (arc tangent), the measured AOA is wrapped.

At this point we can proceed by either unwrapping the measured phase or by "stopping" the fringes. We find the lat- ter to be more robust and easier to implement. Knowing the expected Hydra signal, we proceed by removing the expected unwrapped phase from the complex cross-correlations, i.e. removing the Hydra signal. After this operation, the resulting phase difference is due to the phase offsets of the receiving antennas involved. Figure $2 \mathrm{c}$ shows the results of such procedure (Residual phase). Note that in both cases the residual phases are almost constant between the vertical lines around the transit time $( \pm 5 \mathrm{~min})$, with a very small variance in electrical degrees (less than $10^{\circ}$ ). The phase differences between pairs are found by averaging $10 \mathrm{~min}$ around the transit time. Then the phase offsets of individual receivers are found from the estimated phase differences of the selected pairs.

\subsubsection{Fringe fitting}

Although the previous procedure is very easy to implement, here we present an alternative procedure that allows the estimation of phase offsets making use of not only the phase but also the amplitude of the cross-correlations, therefore providing more degrees of freedom. Since radio stars transit over the site of interest, the receiving signal changes as function of time, namely the signal will be coming from different angles. The expected signal is then given by:

$$
\begin{aligned}
\rho(t)_{i j}= & A_{i j} P_{i}^{1 / 2}\left(\theta_{x}, \theta_{y}\right) P_{j}^{1 / 2}\left(\theta_{x}, \theta_{y}\right) \times \\
& \exp \left[\imath k\left(\Delta x_{i j} \theta_{x}+\Delta y_{i j} \theta_{y}\right)+\imath \Delta_{i j}\right]+C_{i j}+\imath D_{i j}
\end{aligned}
$$

where $P_{i}$ is the antenna pattern of receiving antenna $i, C_{i j}$ and $D_{i j}$ are constants due to a possible cross-talk between signals at antennas $i$ and $j$. Note that the correlation is a function of time, since the location of Hydra changes in time. 
Besides the unknown phase offsets, we are also including the cross-talk terms and amplitudes as unknowns. Making a suitable selection of antenna pairs, we then proceed with a non-linear least-square fitting with the Levenberg-Marquadt algorithm (e.g. Bevington, 1969). Figure 3 shows an example for a pair of antennas with different patterns. The top/bottom panel shows the real/imaginary component of the measured Hydra signal (red/blue, $+/ \times$ ). The fitted signal (black) shows very good agreement with the measured signals, for both antenna pairs selected. Note that for phase calibration purposes, the phase information that we are after is given by the periodicity in time and not by the amplitude, where we observe some small differences that might be due to discrepancies between the actual antenna pattern and the theoretical antenna pattern.

Under ideal conditions, both techniques provide practically the same information. However, when different antennas are used and/or there is cross-talk between channels, then the fringe fitting approach is recommended.

\subsection{Absolute calibration using meteor-head echoes}

Meteor-head echoes come from the plasma in front of the meteoroid entering the Earth's Atmosphere. Using a calibrated three antenna interferometer, the location of a meteorhead echo inside the beam can be determined with a very good precision (e.g. Chau and Woodman, 2004). Figure 4 shows an example of a typical meteor-head echo observed with the Jicamarca high-power large-aperture radar: (a) range vs. time, (b) angular location $\left(\theta_{x}\right.$ vs. $\left.\theta_{y}\right)$, and (c) radial velocity vs. time and signal-to-noise ratio (SNR) vs. time. The quality of the meteor-head data provides excellent location information. Moreover, the measured echo location is verified by comparing the expected SNR (in green) using the theoretical antenna pattern and the measured location information, against the measured SNR (in yellow). In panel c we can see the excellent agreement for this comparison.

Given the good quality of meteor-head echo signals, at Jicamarca we have performed concurrent meteor-head and radar imaging experiments. For the meteor-head experiment, three antenna quarters, that are easier to calibrate, have been used. Then the meteor-head signal is treated as the signal coming from a point target as before. The phases of the receiver channels for the imaging system are estimated by fitting the expected real and imaginary components assuming a coherence of 1 (a good assumption for meteor signals). In the case of the 8 receiver system, there are $28 \times 2$ independent samples to fit for 7 unknown phases. Again, the unknown phases are obtained solving a non-linear least-square fitting using a Levenberg-Marquadt approach. We have found that this approach is more robust than fitting just the phases where there are $2 \pi$ ambiguities associated with the phase estimation. In the latter approach a very good initial guess is needed to reach the global minimum.
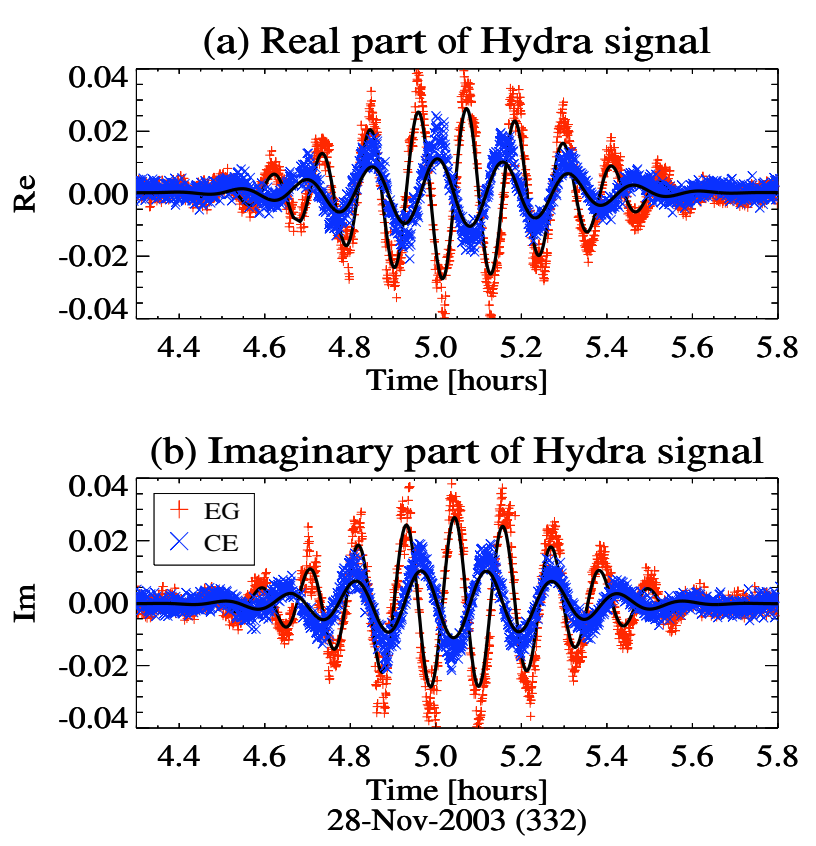

Fig. 3. Fringe fitting. Measured (red $(+)$ and blue $(x)$ and fitted (black) real (top) and imaginary (bottom) Hydra cross-correlation components for selected pair of antennas.

\subsection{Relative calibration using meteor-trail echoes}

Although the procedure using meteor-head echoes works, in practice it can be applied only on very few systems where: (1) the sensitivity is high enough to get reasonable meteorhead echo signals, and (2) a second receiving system with at least three large antennas is available for precise location of the meteor-head echoes.

In this section we present a simple procedure that can be used on smaller radar system. Instead of meteor-head echoes, the procedure relies on the echoes coming from meteor-trails (either specular or non-specular). Again, the signals from meteor-trail echoes are considered to come from a point target, this time with an unknown location. Although the absolute location is unknown, the information can be used for a relative phase calibration.

Our procedure assumes that the meteor signal is coming from a given location (e.g. $\theta_{x}^{\prime}=0$ and $\theta_{y}^{\prime}=0$ ), then we proceed as in the case of the meteor-head echo problem to determine the unknown phase offsets. At this point the imaging system has been relatively calibrated (relative system), and depending on the application one can proceed and use the obtained information, or find a way to get an absolute calibration. As we can see below, our procedure to get the absolute calibration reduces to a mathematical rotation.

In the relative system the phase for the correlation pair $i j$ is

$\phi_{i j}=k\left(\Delta x_{i j} \theta_{x}^{\prime}+\Delta y_{i j} \theta_{y}^{\prime}\right)+\Delta_{i j}^{\prime}$ 
(a)

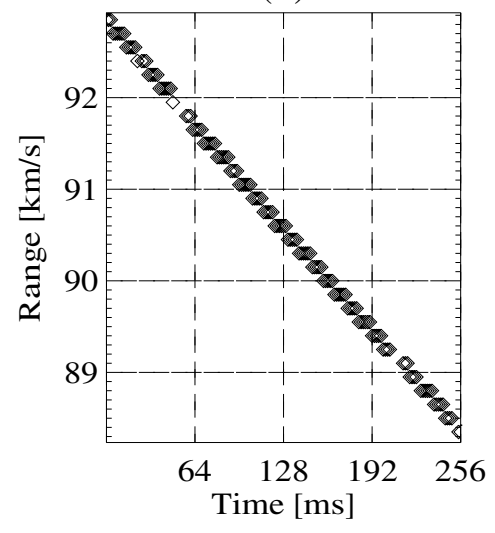

(b)

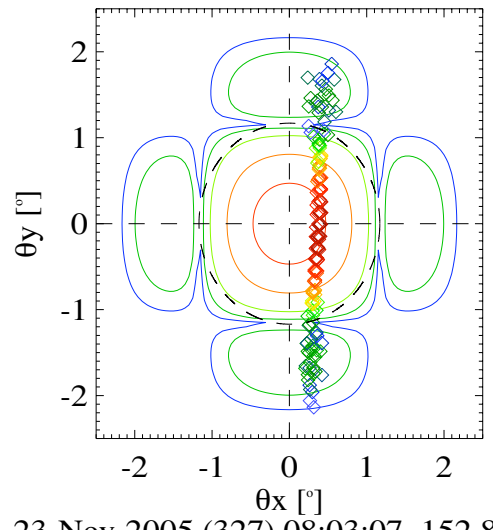

23-Nov-2005 (327) 08:03:07 $152.80 \mathrm{~ms}$

(c)

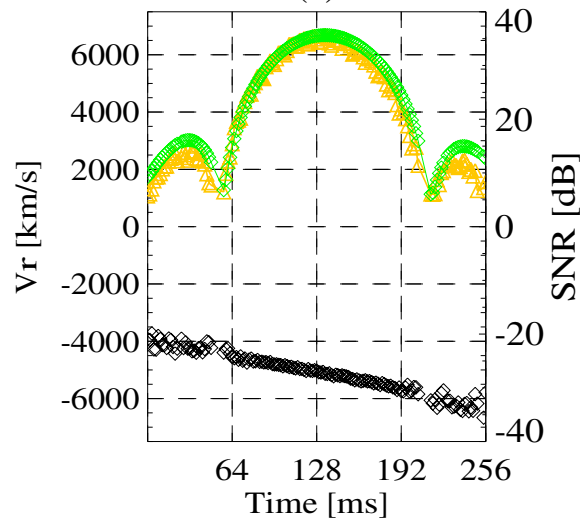

Fig. 4. Example of a meteor-head echo observed at Jicamarca. (a) Range vs. time, (b) $\theta_{x}$ vs. $\theta_{y}$, and (c) radial velocity vs. time and SNR vs. time. The theoretical antenna pattern is indicated in panel (b) for different levels in $\mathrm{dB}$. In panel (c) the measured SNR is indicated in yellow, while the expected relative SNR based on the antenna pattern and angle information, is denoted in green.

where $\theta_{x}^{\prime}=\theta_{x}-\bar{\theta}_{x}, \theta_{y}^{\prime}=\theta_{y}-\bar{\theta}_{y}$, and $\bar{\theta}_{x}, \bar{\theta}_{y}$ are the direction cosines of the meteor location with respect to the absolute system. $\Delta_{i j}^{\prime}$ is the phase offset in the relative system. If one knows the absolute location of the meteor echo $\left(\bar{\theta}_{x}, \bar{\theta}_{y}\right)$, then the new phase offsets of the absolute system $\left(\Delta_{i j}\right)$ can be obtained from

$\Delta_{i j}=\Delta_{i j}^{\prime}-k\left(\Delta x_{i j} \bar{\theta}_{x}+\Delta y_{i j} \bar{\theta}_{y}\right)$

This procedure should work as long as the electrical and geometrical centers of the antennas remain the same for all the direction cosines of interest. Note that, in general this procedure will not work for non-coplanar antennas. It will be approximately true so long as the targets used for calibration are nearly collocated.

In the case of Jicamarca, we find the absolute location by using the known mean characteristics of EEJ echoes, i.e. after a few minutes of averaging, its main location is given as the locus of perpendicularity in the north-south direction and the main antenna position in the East-West location. The averaged location of the EEJ using the relative phases obtained from the meteor-trail calibration is found using three noncollinear antennas (e.g. the three antennas furthest to the left in Fig. 1). Comparing the measured location against the expected location, we find $\bar{\theta}_{x}, \bar{\theta}_{y}$.

The comparison of this technique against the use of Hydra or meteor-head echoes is very good. Instead of showing results of the comparisons, in Fig. 5, we show examples of EEJ images obtained using the meteor-trail procedure. In this example, we can clearly see the good quality of EEJ signals in conjunction with an example of a well defined meteor-trail echo. As in our previous papers showing radar images of equatorial ionospheric irregularities, each frame shows the altitude vs. zonal structure of irregularities inside the illuminated volume. The lightness, hue, and saturation of the im- age pixels reflects the intensity, Doppler shift, and spectral width of the corresponding echoes. The EEJ images shows clearly the expected kilometer scale waves at the top for typical daytime EEJ echoes (e.g. Hysell and Chau, 2002). Each image has been obtained with a $\sim 5 \mathrm{~s}$ integration time ("exposure"). The meteor-trail echo is well defined with a resolution limited by the angular and altitude resolution of the radar system. An un-calibrated system or poorly calibrated would have produced either ghost images of the meteor trails or wider trails.

\subsection{Systematic calibration using a radio beacon}

Until now, all the procedures presented are applicable on small portions of data. If the phases of the system remain the same for the duration of a given campaign, then any of the calibration procedures described in this paper or others listed in the introduction, can be applied at any time. However, on some installations such phases do not remain the same, either due to constant changes in the system or to a poor installation (e.g. receiving cables not protected for temperature variations). At Jicamarca, both situations are encountered, therefore both short term and long term phase changes are expected.

In order to be able to perform imaging experiments at Jicamarca more often and more reliably, we are using a radio beacon signal that emits a very low power pulsed signal from one of the hills surrounding Jicamarca (indicated with a yellow circle in Fig. 1). Such signal is synchronized with the main transmitter system, allowing the location of the radio beacon signal at desired ranges, where ionospheric signals are not expected. Moreover, the beacon signal is narrow band inside the bandwidth of the main system. In principle, one could take into account the exact geometry of the configuration, 

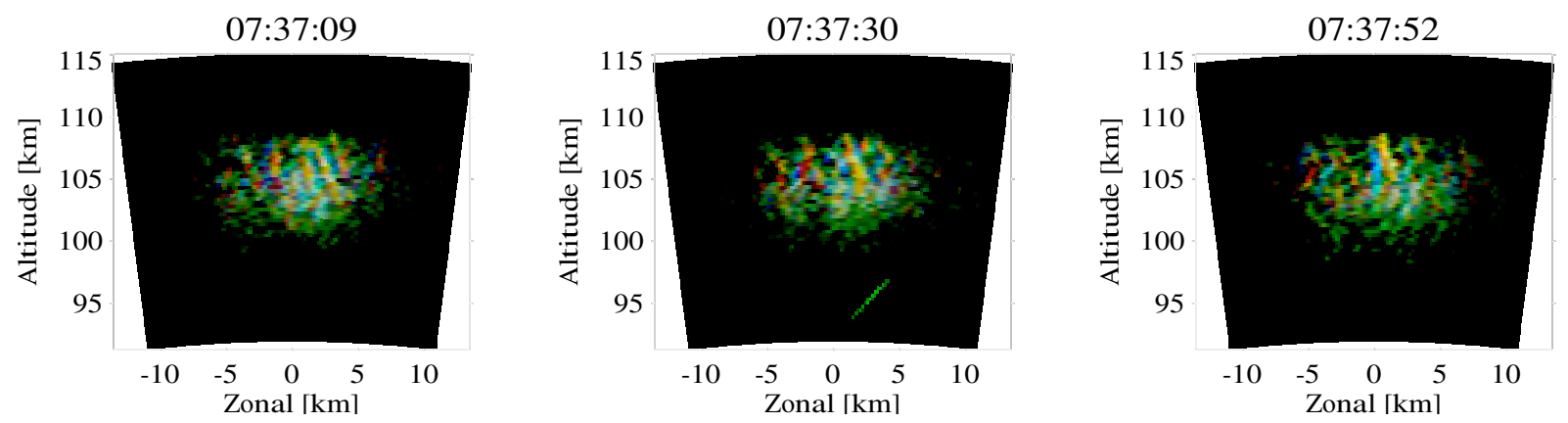

Fig. 5. Radar images of EEJ events. The phase calibration has been done using a meteor-trail echo and the mean EEJ characteristics. Each frame has been obtained with $5 \mathrm{~s}$ exposure.

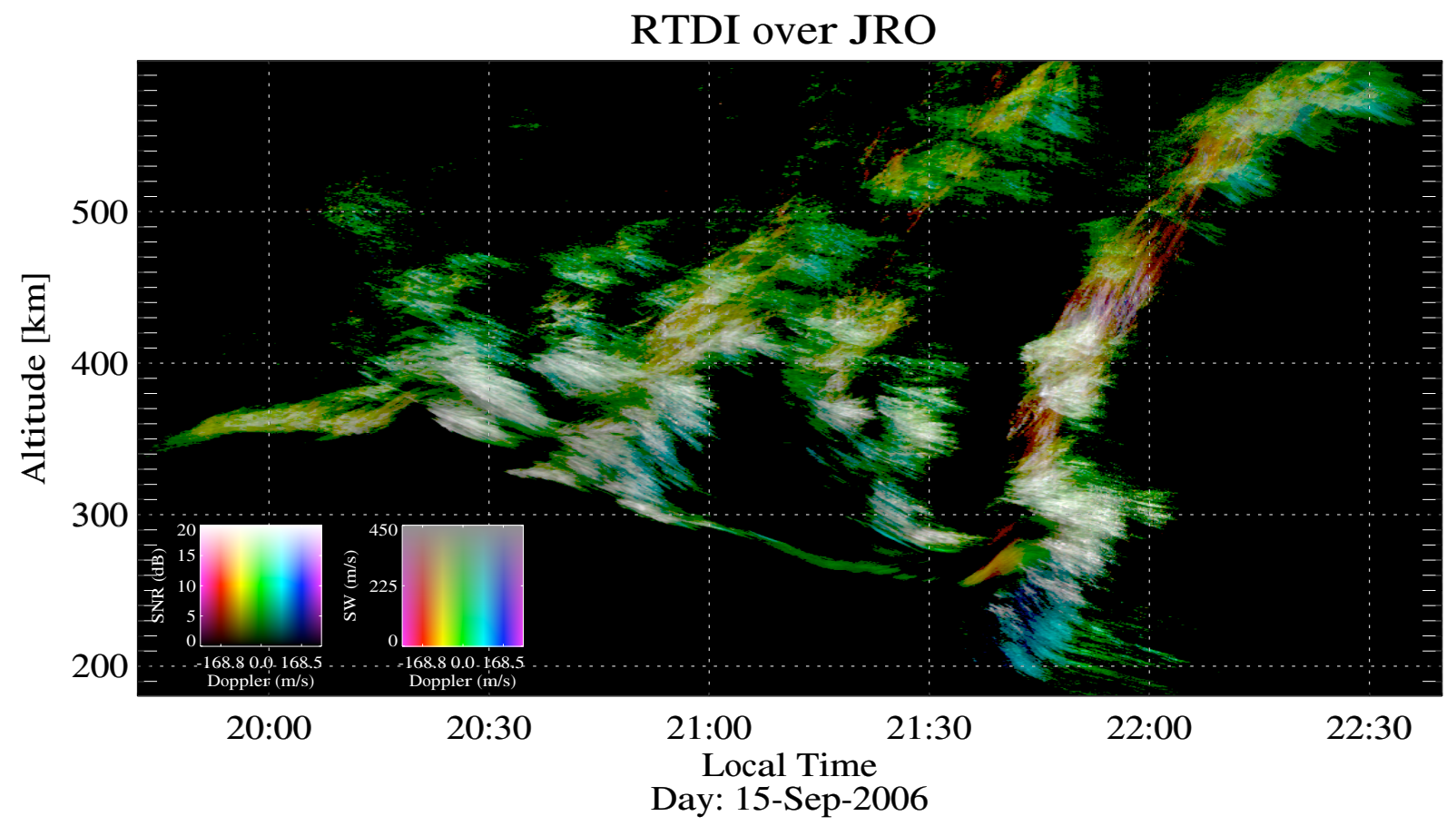

Fig. 6. Modified range-time intensity plot using Doppler information (RTDI). The lightness, hue, and saturation of the image pixels reflects the SNR, Doppler shift, and spectral width of the corresponding echoes. The legend on the left represents the SNR vs. Doppler color map for a saturation value of $100 \%$. The legend on the right represents the spectral width vs. Doppler color map for a $50 \%$ of lightness.

i.e. beacon position and antenna centers, to obtain the expected phase changes, particularly since the radio beacon is inside the near field of the array configuration. However, to avoid phase changes due to multi-path from the surrounding mountains and possible differences between the far-field and near-field electric centers (since the radio beacon signal is coming from low elevation angles), we have found that an empirical approach is more reliable.

Our empirical approach consists of:

- Calibrating a given imaging configuration with one of the aforementioned techniques and obtaining the calibrated phases $\left(\delta_{c}\right)$.
- Running (simultaneously) the radio beacon and obtaining the the phase offsets from a beacon signal $\left(\delta_{b}\right)$.

- Obtaining an empirical phase difference between the calibrated values and the beacon values $\left(\delta_{c b}=\delta_{c}-\delta_{b}\right)$.

- Carrying out this procedure for each of the receiving channels.

As long as the beacon and the antennas do not change position, $\delta_{c b}$ should remain constant. Therefore, by measuring the beacon phase, one can get the desired calibrated phase at any time, since the beacon signal will take into account 

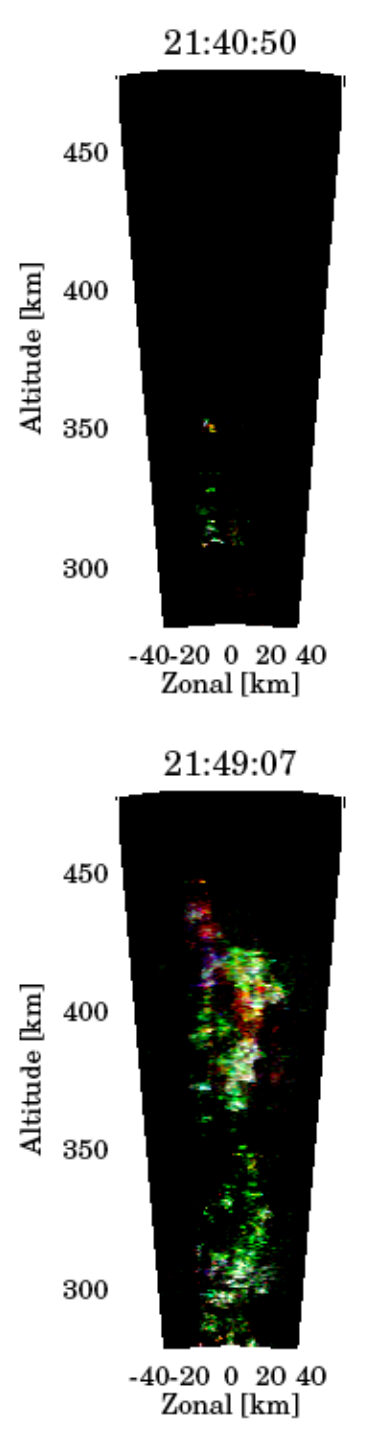
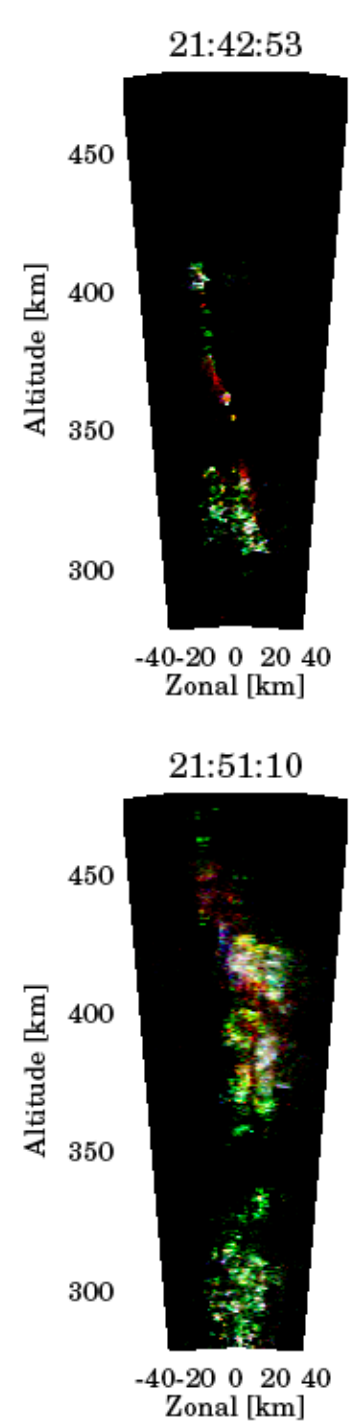
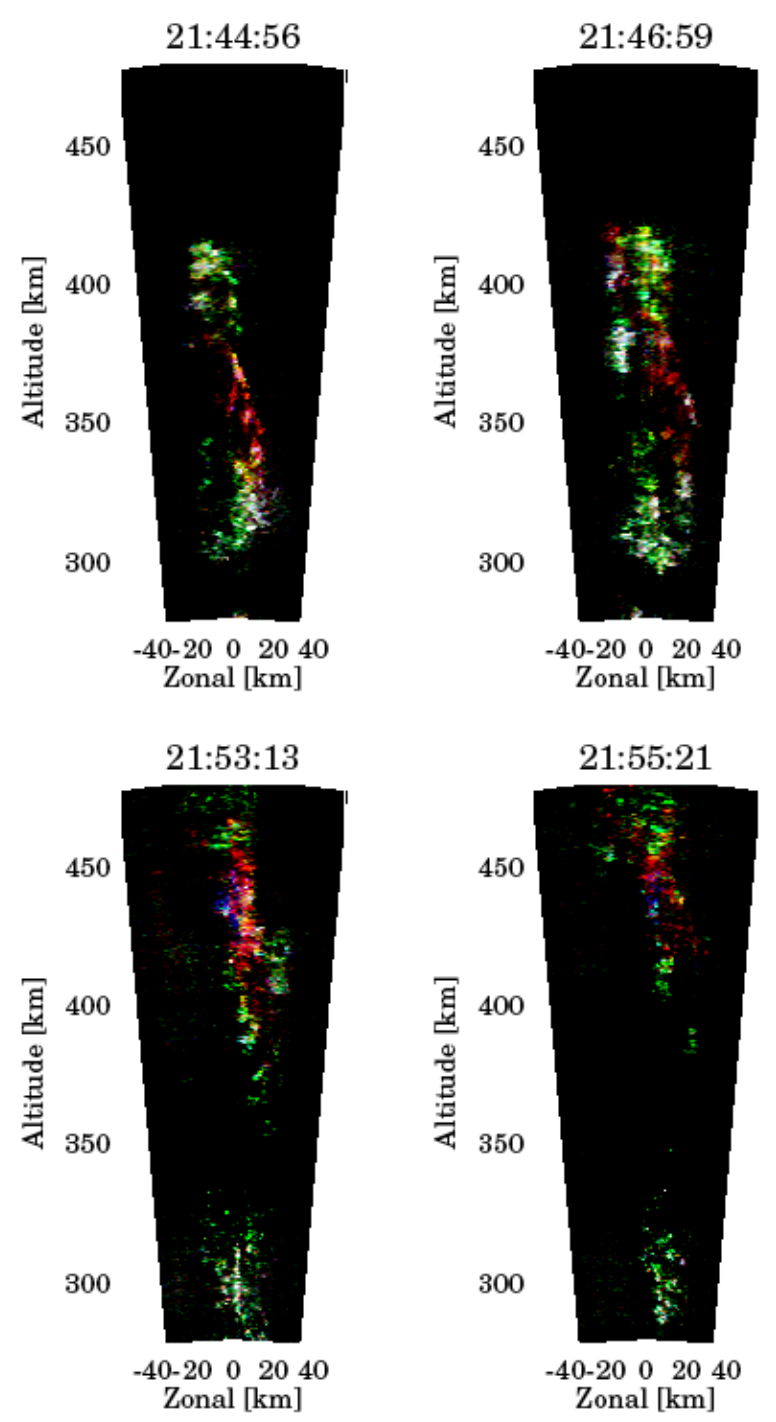

Fig. 7. Example of ESF images showing depleted channels pinching off a bottomside layer. Each frames has been obtained with $10 \mathrm{~s}$ exposure. Note that the red depleted channels move independently of the surrounding regions that in general drift horizontally.

any of the phase offsets described above, both short and long term.

In the next section, we present ESF imaging results obtained with the beacon approach. We have been applying this approach successfully at different times and with different hardware (receivers, filters, receiving lines, etc.), but the same antenna configuration. We are currently using a similar approach for a recent specular meteor system installed at Jicamarca in collaboration with the Australian company ATRAD. The phases obtained from the beacon approach are in excellent agreement with in-situ calibration phases and the phases from the self-calibration approach of Holdsworth et al. (2004b).

\section{Modified RTI map and equatorial spread F results}

Although the main goals of this work are not directly related to geophysics, in this section we present geophysical results related to ESF. Such features can be observed and described due to the employment of radar imaging techniques and in turn by the technical improvements described in this paper.

In Fig. 6 we present an alternative approach to the classical range-time intensity (RTI) maps used in previous ESF works (e.g. Woodman and La Hoz, 1976). Instead of using a false color scheme, where a given intensity value is associated with an arbitrary color (or gray tone), in this modified RTI, color represents Doppler information. The lightness, hue, and saturation of the image pixels reflects the intensity, Doppler shift, and spectral width of the corresponding 
echoes. This new RTDI (Range-time Doppler Intensity) map is obtained by combining the intensity maps of three different Doppler bins, i.e. red (around $-170 \mathrm{~m} / \mathrm{s}$ ), green (around $0 \mathrm{~m} / \mathrm{s}$ ), and blue (around $170 \mathrm{~m} / \mathrm{s}$ ).

The RTDI map still has limitations of a slit camera, where spatial and time ambiguities could create distortions and complicate the interpretation of the results (e.g. Woodman, 1997). However, we think that the RTDI map provides much more information than a typical RTI. For example, we can identify depleted channels with completely different radial velocities than the background (red structures around 21:40 LT, see below). We expect to find other uses of the proposed RTDI, particularly for monostatic radar systems, but such efforts will be left for future work.

To clarify the interpretation of the event around 21:40 LT, in Fig. 7 we show two dimensional (altitude vs. zonal distance) images. Each image has been obtained with a $\sim 10 \mathrm{~s}$ integration time. As in the case of the RTDI map, lightness, hue, and saturation of the image pixels reflects the intensity, Doppler shift, and spectral width of the corresponding echoes, using the same color legend of the RTDI map. The same convention has been used in previous radar imaging work at Jicamarca (e.g. Hysell and Chau, 2002; Chau and Hysell, 2004; Hysell and Chau, 2006). Time progresses from left to right and from top to bottom at $\sim 2$ min intervals. We can clearly identify the narrow depleted channel in red, moving independently of its surrounding regions, including the bottom layer where it initiates. This channel appears to have pinched off from the bottomside, moving away from the radar at $\sim 150-200 \mathrm{~m} / \mathrm{s}$. It started around $320 \mathrm{~km}$ around $21: 41 \mathrm{LT}$ and continue going up to $\sim 500 \mathrm{~km}$ around 21:55 LT. Note that coexisting with the narrow depleted red channel there are surrounding regions drifting horizontally independent of the channel. Similar radar observations, including the bifurcation of intermediate- and largescale plasma depletions has been reported and interpreted by Hysell (1999) and Hysell (2000).

Pinching was first hypothesized by Aggson et al. (1993) who presented electric field data data from San Marco spacecraft showing the horizontal walls of a channel moving toward one another. Although the ionospheric flow is very nearly incompressible, diffusion could fill a depleted channel if it were to become sufficiently narrow through its own evolution. Hysell (1999) performed linear nonlocal analyses of pinching and bifurcating depletions and found that both could be explained in terms of secondary instabilities. For the case of pinching, the equilibrium configuration considered was that of depleted plasma rushing through a twodimensional nozzle channel between high plasma density walls. More details about the pinching and bifurcation events can be found in Hysell (2000).

Besides the pinching event, there is much more information on the radar images associated with the observations associated with the RTDI event of Fig. 6. In the supplemental material we present two dimensional movies showing the rich morphology and dynamics of ESF irregularities inside the illuminated beam. Here is a brief description of the results in the supplemental material:

- RTDI and frames (http://www.ann-geophys.net/26/ 2333/2008/angeo-26-2333-2008-supplement.zip: movie1.mpg). In this animated file we show the typical slit camera nature of RTDI plots. In addition we are including the image frame that corresponds to the time indicated by the vertical red line over the RTDI. The movie shows the evolution of backscatter from bottom-type layers, bottomside layers and topside plumes, drifting and rising through the ionospheric regions illuminated by the Jicamarca radar. Contrary to previous ESF images, the results presented here have been taking during solar minimum conditions; therefore, plumes are observed at lower altitudes. Moreover topside images are less diffuse and less fuzzy than previous topside images, given that amplitude and phase scintillations are expected to be less.

- Bottomside and topside plumes (http: //www.ann-geophys.net/26/2333/2008/

angeo-26-2333-2008-supplement.zip: movie2.mpg). In this movie we show frames every $10 \mathrm{~s}$ between $280 \mathrm{~km}$ and $480 \mathrm{~km}$ and between 19:50 LT and 21:36 LT. During the first $20 \mathrm{~min}$, we can clearly observe the shear flow around $360 \mathrm{~km}$ and the generation of secondary instabilities. The bottom region drifting westward while the upper regions drifting eastward at times and altitudes when the E-region and F-region dynamos are still competing. Later, we can observe the evolution and occurrence of all kinds of intermediate and large scale instabilities, some of them bifurcating and pinching off from the bottom regions.

- Pinching event (http://www.ann-geophys.net/26/ 2333/2008/angeo-26-2333-2008-supplement.zip:

movie3.mpg). This movie also contains frames every $10 \mathrm{~s}$ but this time between $200 \mathrm{~km}$ and $400 \mathrm{~km}$ and between 21:33 LT and 22:02 LT. Besides a variety or intermediate and large scale structures, we can clearly identify a very narrow depleted channel pinching around $250 \mathrm{~km}$ and rising as high as $500 \mathrm{~km}$ (not shown in the animation, but seen in the movie1.mpg file). This animation complements the frames shown in Fig. 7. Contrary to the frames in the main text, in the movie one can clearly observe that the surrounding regions are moving independently of the depleted channel, moreover, there are regions even drifting down indicated with the blue regions.

For more details on the interpretation and instabilities behind the animated results, the reader should consult Hysell (1999) and Hysell (2000). We suggest using a video player where the playing speed can be controlled, for example VLC 
a cross-platform media player that can be downloaded from http://www.videolan.org.

\section{Concluding remarks}

We have presented three non-conventional phase calibration approaches for radar systems using multiple-receiver capabilities. Except for the approach using meteor-head echoes, we think the other approaches can be easily implemented at other sites, particularly, where long baselines are used, configurations are constantly changing, and/or reduced cost on receiving installation is required (e.g. to avoid running all receiving cables underground to keep temperature stability).

Depending on the approach selected, we have used either the phase or complex information. Using Hydra, both options work under ideal conditions. However, when there are imperfections in the system (e.g. cross-talk between channels), using the complex (phase and amplitude) information is more reliable. In the case of meteor echoes (for both head and trails), solving the non-linear least square fitting for the real and imaginary components is more robust than working with just the phases.

To complement the absolute and relative phase calibration approaches, we have shown that the radio beacon approach works well at Jicamarca. Using the pulsed signal from a radio beacon, a given receiving configuration, needs to be calibrated only once, as long as the beacon remains in the same position. Calibrating a given system with one of the techniques described above, an empirical phase difference with respect to the beacon signal can be obtained and later use in operational experiments. In principle, even pulse-to-pulse corrections could be applied. Such approach is currently being used successfully for all imaging experiments to study the field aligned irregularities at Jicamarca. This technique is also being used to calibrate an all-sky meteor system.

Instead of making a statistical study of the performance of the techniques, we have shown the good performance of the phase calibration techniques presenting EEJ, meteor trail and ESF images. In the case of the meteor trail and EEJ, a bad calibration will be easily identified.

We have also shown ESF results and introduced a new RTI map that uses the Doppler information (RTDI) in a analogous manner to the radar images. We expect that the new RTDI will help identify peculiar events previously hidden in usual RTI maps. For example, we have been able to spot events pinching off from bottomside regions. Such events have been corroborated by the radar images, that show the very narrow depleted regions with a distinct Doppler information, evolving inside the illuminated beam. We expect the RTDI maps will help us identify pinching events in monostatic maps (or using only two-antenna configurations) to study how prevalent pinching is in ESF.

Finally, since radar imaging helps us to extract information about the spatial structure of the irregularities and the flow, we expect to conduct this type of experiments in long-term basis at Jicamarca (JULIA-like experiments). Besides implementing the recent improvements proposed by Hysell and Chau (2006), we are confident that the phase calibration approaches described in this paper will be instrumental in such efforts. These future observations will be useful in our efforts of finding evidence for the seeding of ESF irregularities (e.g. Hysell et al., 2004a), comparison with airglow images obtained with narrow-field of view cameras north and south of Jicamarca (J. Makela, private communication, 2006), and comparisons with recent numerical simulations (e.g. Huba and Joyce, 2007).

Acknowledgements. We wish to thank W. Coles, D. Holdsworth, and R. Woodman for their insightful suggestions on the different phase calibration procedures implemented in this work. The Jicamarca Radio Observatory is a facility of the Instituto Geofísico del Perú operated with support from the NSF Cooperative Agreement ATM-0432565 through Cornell University.

Topical Editor U.-P. Hoppe thanks G. Hassenpflug and another anonymous referee for their help in evaluating this paper.

\section{References}

Aggson, T. T., Hanson, W. B., Herraro, F. A., Maynard, N. C., Pfaff, R. F., Saba, J. L., and Tsunoda, R. T.: Equatorial electric field observations, Adv. Space Res., 13, 271-291, 1993.

Bahcivan, H., Hysell, D. L., Larsen, M. F., and Pfaff, R. F.: The $30 \mathrm{MHz}$ imaging radar observatipns of auroral irregularities during the JOULE campaign, J. Geophys. Res., 110, A05307, doi:10.1029/2004JA010975, 2005.

Bahcivan, H., Hysell, D. L., Lummerzheim, D., Larsen, M. F., and Pfaff, R. F.: Observation of colocated optical and radar aurora, J. Geophys. Res., 111, A12308, doi:10.1029/2006JA011923, 2006.

Bevington, P. R.: Data Reduction and Error Analysis for the Physical Sciences, McGraw Hill, 1969.

Chau, J. L. and Balsley, B. B.: A statistical comparison of VHF techniques to study clear-air vertical velocities in the lower atmosphere using the Jicamarca radar, Radio Sci., 33, 1565-1583, 1998.

Chau, J. L. and Hysell, D. L.: High altitude large-scale plasma waves in the equatorial electrojet at twilight, Ann. Geophys., 22, 4071-4076, 2004, http://www.ann-geophys.net/22/4071/2004/.

Chau, J. L. and Woodman, R. F.: Three-Dimensional Coherent Radar Imaging at Jicamarca: Comparison of Different Inversion Techniques, J. Atmos. Sol. Terr. Phys., 63, 253-261, 2001.

Chau, J. L. and Woodman, R. F.: Observations of Meteor-Head Echoes Using the Jicamarca $50 \mathrm{MHz}$ Radar in Interferometer Mode, Atmos. Chem. Phys., 4, 511-521, 2004, http://www.atmos-chem-phys.net/4/511/2004/.

Farley, D. T., Ierkic, H. M., and Fejer, B. G.: Radar interferometry: A new technique for studying plasma turbulence in the Ionosphere, J. Geophys. Res., 86, 1467-1472, 1981.

Holdsworth, D. A., Reid, I. M., and Cervera, M. A.: Buckland park all-sky interferometric meteor radar, Radio Sci., 39, RS5009, doi:10.1029/2003RS003014, 2004a. 
Holdsworth, D. A., Tsutsumi, M., Reid, I. M., Nakamura, T., and Tsuda, T.: Interferometric meteor radar phase calibration using meteor echoes, Radio Sci., 39, RS5012, doi:10.1029/2003RS003026, 2004b.

Huba, J. D. and Joyce, G.: Equatorial spread F modeling: Multiple bifurcated structures, secondary instabilities, large density 'biteouts', and supersonic flows, Gephys. Res. Lett., 34, L07105, doi:10.1029/2006GL028519, 2007.

Hysell, D. L.: Radar Imaging of Equatorial $F$ Region Irregularities with Maximum Entropy Interferometry, Radio Sci., 31, 15671578, 1996.

Hysell, D. L.: Imaging Coherent Scatter Radar Studies of Equatorial Spread F, J. Atmos. Sol. Terr. Phys., 61, 701-716, 1999.

Hysell, D. L.: An Overview and Synthesis of Plasma Irregularities in Equatorial Spread F, J. Atmos. Sol. Terr. Phys., 62, 10371056, 2000.

Hysell, D. L. and Burcham, J. D.: JULIA Radar Studies of Equatorial Spread F, J. Geophys. Res., 103, 29 155-29 167, 1998.

Hysell, D. L. and Chau, J. L.: Imaging Radar Observations and Nonlocal Theory of Large-Scale Waves in the Equatorial Electrojet, Ann. Geophys., 20, 1167-1179, 2002, http://www.ann-geophys.net/20/1167/2002/.

Hysell, D. L. and Chau, J. L.: Optimal aperture synthesis radar imaging, Radio Sci., 41, RS2003, doi:10.1029/2005RS003383, 2006.

Hysell, D. L. and Woodman, R. F.: Imaging Coherent Backscatter Radar Observations of Topside Equatorial Spread $F$, Radio Sci., 32, 2309-2320, 1997.

Hysell, D. L., Chau, J. L., and Fesen, C. G.: Effects of Large Horizontal Winds on the Equatorial Electrojet, J. Geophys. Res., 107(A8), 1214, doi:10.1029/2001JA000217, 2002.

Hysell, D. L., Chun, J., and Chau, J. L.: Bottom-type scattering layers and equatorial spread $F$, Ann. Geophys., 22, 4061-4069, 2004a, http://www.ann-geophys.net/22/4061/2004/.
Hysell, D. L., Larsen, M. F., and Zhou, Q. H.: Common volume coherent and incoherent scatter radar observations of mid-latitude sporadic E-layers and QP echoes, Ann. Geophys., 22, 32773290, 2004b, http://www.ann-geophys.net/22/3277/2004/.

Jones, J., Webster, A. W., and Hocking, W. K.: An improved interferometer desing for use with meteor radars, Radio Sci., 33, 55-66, 1998.

Kudeki, E. and Sürücü, F.: Radar Interferometric Imaging of FieldAligned Plasma Irregularities in the Equatorial Electrojet, Geophys. Res. Lett., 18, 41-44, 1991.

Kudeki, E., Farley, D. T., and Fejer, B. G.: Long Wavelength Irregularities in the Equatorial Electrojet, Geophys. Res. Lett., 9, 684-687, 1982.

Palmer, R. D., Vangal, S., Larsen, M. F., Fukao, S., Nakamura, T., and Yamamoto, M.: Phase calibration of VHF spatial interferometry radars using stellar sources, Radio Sci., 31, 147-156, 1996.

Palmer, R. D., Gopalam, S., Yu, T. Y., and Fukao, S.: Coherent Radar Imaging Using Capon's Method, Radio Sci., 33, 15851598, 1998.

Taylor, G. B., Perley, R. A., and Carilli, C. L. (Eds.): Synthesis Imaging in Radio Asronomy II, 704 p., Astronomical Society of the Pacific, 1999.

Valentic, T. A., Avery, J. P., Avery, S. K., and Livingston, R. C.: Self-survey calibration of meteor radar antenna arrays, IEEE Trans. Geosci. Remote Sensing, 35, 524-531, 1997.

Woodman, R. F.: Inclination of the geomagnetic field measured by an incoherent scatter technique, J. Geophys. Res., 76, 178-184, 1971.

Woodman, R. F.: Coherent Radar Imaging: Signal Processing and Statistical Properties, Radio Sci., 32, 2373-2391, 1997.

Woodman, R. F. and La Hoz, C.: Radar Observations of $F$ Region Equatorial Irregularities, J. Geophys. Res., 81, 5447-5466, 1976.

Woodman, R. F., Veliz, O., Aquino, F., Villanueva, F., and Sarango, M.: Radio Star Scintillations at $50 \mathrm{MHz}$ Produced by Electrojet Irregularities, paper presented at the Ninth Int. Symposium on Equatorial Aeronomy, Bali, Indonesia, 1995. 